\title{
Entwicklung eines Geschäftmodells
}

\author{
Christian Halama, Nico Venske
}

\section{Zusammenfassung}

Durch die Anbindung von diversen Geräten, wie z. B. Mobiltelefonen, PDAs (Personal Digital Assistants), an das Internet und Technologien, wie WAP (Wireless Application Protocol), GPRS (General Packet Radio Service) und in weiterer Folge UMTS (Universal Mobile Telecommunications System), wird der orts- und zeitunabhängige Zugriff auf Daten und Informationen ermöglicht. Viele Unternehmen wollen an diesen Chancen des M-Commerce partizipieren und tätigen deshalb ernorme Investitionen. Wenn diese Investitionen rentabel sein sollen, dann ist eine realistische Betrachtung der Marktsituation, der Geschäftsmodelle und der Erfolgsfaktoren im M-Commerce unumgänglich.

Im Masterstudiengang »Telematik « wurde im Wintersemester 2006/07 das Fach Mobile Commerce unterrichtet. In der Vorlesung wurden von Prof. Dr. Bernd Eylert die zur Entwicklung eines Geschäftsmodells nötigen Schritte und Vorraussetzungen behandelt. In der vorliegenden Arbeit wurde das vermittelte Wissen auf ein neuartiges Kameraüberwachungssystem für Wohnungen angewandt. In Zusammenarbeit mit Dr. Reiner Krämer (T-Systems) wurde eine Aufgabenstellung definiert, die zwei Schwerpunkte enthielt. Zum einen sollte eine Marktanalyse durchgeführt werden. Dafür wurden Konkurrenzprodukte und dessen Hersteller untersucht, deren Funktionen analysiert und die Preise ermittelt. Zum anderem sollte ein Businessmodell erstellt werden. Es bezieht sich auf die UMTS-Variante des Überwachungssystems und enthält die Betrachtung der technischen Lösung und die Berechnung des Businessmodells.

\section{Abstract:}

The connection of miscellaneous devices like portable phones, PDAs (Personal Digital Assistants) to the internet with technologies like WAP (Wireless Application Protocol), GPRS (General Packet Radio Services) and in further scope UMTS (Universal Mobile Telecommunications System) makes it possible to access data and information time independent. Many business players want to participate at these new chances of the M-Commerce and therefore they encourage investments. If these investments should be profitable, a realistic contemplation of the market situation, the business models and the success-factors are absolutely necessary in the view of M-Commerce.

In the master study of »telematics « one of the subjects is Mobile Commerce. In the lecture from Prof. Dr. Bernd Eylert the development of a business model with all steps was mediated. In the present work, the mediated knowledge was applied to a new camera supervision system for apartments. In cooperation with Dr. Reiner Krämer (T-Systems) the design, which two main focuses contains, was defined. First a market analysis should be done. Steps were the finding of competition products and its manufacturer, analysing the functions and compare the prices in the market. In the second part, a business model should be produced. Also the UMTS variation of the supervision system, the contemplation of the technical solution and the calculation of the business model were parts of this.

\section{Ausgangssituation und Begriffsdefinition}

Der Markt für Informationstechnologien ist in den letzten Jahren stark gewachsen. Durch die globale Vernetzung der wirtschaftlichen Beziehungen und Kommunikationswege ergeben sich für die Beteiligten im Geschäftsleben sowohl große Chancen als auch Risken. Neben Electronic Commerce ist in letzter Zeit immer öfter der Begriff des Mobile Commerce in diversen Medien wahrzunehmen. Was ist unter diesen Begriffen zu verstehen?

Unter Electronic Commerce (E-Commerce), was frei übersetzt soviel wie »Elektronischer Handel« bedeutet, ist der Austausch von Gütern und Dienstleistungen über Rechnernetze, insbesondere das Internet, zu verstehen.
Ein Synonym dafür ist das Electronic Business (E-Business), was für »Elektronische Geschäfte«steht.

Schwieriger verhält es sich mit dem Begriff Mobile Commere (M-Commere), da er in der Literatur und Praxis unterschiedlich abgegrenzt wird. Nach Eylert versteht man unter Mobile Commerce jegliche Art von geschäftlichen Transaktionen, bei der die Transaktionspartner mobile elektronische Kommunikationstechniken, wie z. B. Mobilfunk, Wireless Lan oder Bluetooth, nutzen. Nach Wirtz wird unter dem Begriff Mobile Commerce die Anbahnung sowie die teilweise, respektive vollständige Unterstützung, Abwicklung und Aufrechterhaltung von Leistungsaustauschprozessen mittels elektronischer Netze und mobiler Zugangsgeräte verstanden. 


\section{Unternehmensprofil T-Systems}

T-Systems ist ein international operierender Dienstleister für Informations- und Kommunikationstechnologie (ICT). Das Unternehmen war bis Herbst 2007 neben T-Online, T-COM und T-Mobile eine der vier Säulen der Deutschen Telekom AG mit Sitz in Frankfurt am Main. Insgesamt beschäftigte T-Systems 2007 in 24 Ländern ca. 52.000 Mitarbeiter. Die Ausrichtung als ICT-Anbieter hat den Hintergrund, dass die Grenze zwischen der Informationstechnik (IT) und der Telekommunikation (TK) langsam verwischt und die Bereiche immer stärker verschmelzen.

Durch das angebotene Leistungsspektrum versteht sich das Unternehmen als »One-Stop-Shop« für die gesamte IT-Wertschöpfungskette ihrer Kunden zu positionieren, die von Telekommunikationsdienstleistungen bis hin zu IT-Services alles beinhaltet. Die Fachkunde des Unternehmens baut auf der Erfahrung, dem Spezialistenwissen und dem Streben nach »TopPerformance«auf.

\subsection{Marktauftritt und Leistungsfähigkeit}

»Nur starke Marken setzen sich auf dem ICT-Markt durch «. Mit dieser Aussage ist T-Systems Anfang 2005 nach der strategischen Neuausrichtung der Deutschen Telekom AG als Marke des Konzerns neu gestartet. Als großer »Startup« im ICT-Bereich hat sich T-Systems auf der Basis strategischer Überlegungen und fundierter Marktstudien für einen klaren und konsistenten Auftritt mit der Metapher »High-Tech Segeln « entschieden. Auf diese Weise erreicht das Unternehmen kontinuierlich eine hohe Wiedererkennung und einen einzigartigen »Fit « von Bild- und Wertewelt für die Marke T-Systems. Als Geschäftskundenmarke der Deutschen Telekom ist das Unternehmen eng mit der T-Marke verzahnt und differenziert sich über die Zielgruppe und deren Bedürfnisse und Interessen. Ein Beispiel für die Leistungsfähigkeit von T-Systems ist das Argus-Überwachungssystem. Das System wurde auf Wunsch des Unternehmens durch einen Zulieferer hardwaretechnisch entwickelt und bereitgestellt. Als Gegenleistung bot T-Systems dem Sicherheitsunternehmen einen Vertrag als fester Zulieferer, was besonders für kleine Unternehmen einen großen Erfolg darstellt. So können diese Unternehmen einerseits damit werben, für T-Systems tätig zu sein, und andererseits feste Aufträge einplanen. Die softwareseitige Anbindung des Überwachungssystems entwickelte T-Systems selbst.

\subsection{Marktauftritt Argus}

Wie bereits angedeutet, wird das Argus-Überwachungssystem von T-Systems nicht direkt an den Endkunden vertrieben. Das Sicherheitssystem wird sich dem privaten Endkunden am Markt über Internet-Service-Provider und andere Sicherheitsunternehmen präsentieren. Das sind die Geschäftspartner der T-Systems. Bei erfolgreicher Umsetzung des Sicherheitssystems steht T-Online als Vertriebspartner bereits jetzt fest. Weitere Gespräche mit Freenet, $1 \& 1$ u. v. m. sind geplant. Die Provider werden versuchen, einen neuen Zusatzdienst zu verkaufen, mit dem sie Neukunden gewinnen können.

Durch den erhöhten Traffic auf ihren Datenleitungen könnten auch Nutzer von Zeit- oder Volumentarifen zum Wechsel auf eine Flatrate überzeugt werden. Für Sicherheitsunternehmen bieten sich neue Möglichkeiten durch den Vertrieb von Low Cost-Sicherheitssystemen, für die sie einen »Rundum-Service« anbieten können. Dieser Service könnte z. B. die Beschaffung des Breitbandanschlusses, die Montage und Einstellung des Gerätes und einen ständigen Kundenservice beinhalten.

\section{Wettbewerb}

Um das Überwachungssystem Argus im Sicherheitsmarkt richtig positionieren zu können, müssen Konkurrenzprodukte und dessen Hersteller genau analysiert werden. Dazu wurden alle relevanten Überwachungssysteme, welche mit Argus vergleichbar sind, ermittelt und anhand von Funktionen und Parametern einander gegenübergestellt. Von einem Hersteller werden meist mehrere Produkte mit unterschiedlichen Leistungsmerkmalen angeboten. Aus der Vielfalt aller ermittelten Produkte sind die Leistungsfähigsten mittels einer detaillierten Untersuchung weiter zu analysieren.

Der Detailuntersuchung (siehe Tab. 1) ist neben den ausgesuchten Geräten noch eine Kombination aus einer einfachen Kamera und der Überwachungssoftware go1984 hinzugefügt. go1984 ist eine professionelle, für Netzwerkanwendungen konzipierte Überwachungssoftware, mit der es möglich ist, verschiedene Kameras zu verwalten, zu steuern und zu überwachen. Eine Bewegungserkennung und Alarmierung ist ebenfalls integriert und lässt sich komfortabel bedienen. Nachteilig ist bei der Software, dass ein PC im Netzwerk notwendig ist.

Zusammenfassend lässt sich sagen, dass im Bereich der Netzwerkanbindungen (W-LAN/ LAN) der Kameras ein breites Spektrum an Endgeräten existiert. Die untersuchten Geräte bieten alle wichtigen Grundvoraussetzungen, um eine Videoüberwachung von Räumen durchzuführen. Sie unterscheiden sich nur in der Qualität (Methodik) der Bewegungserkennung sowie in der Integration von weiteren Sensoren und der Anschlussmöglichkeit externer Sensorik. Durch die Vergabe einer dynamischen DNS kann man aus dem kompletten WWW via Browser auf die Kamera zugreifen. Das ermöglicht einen einfachen und unkomplizierten Zugang zu Livedaten von der Kamera. Der Wettbewerberbereich für Lösungen mittels Kommunikation über Mobilfunk ist sehr übersichtlich. Lediglich die Kamera PT-6 von Nokia erfüllt die gewünschten Anforderungen. Die Kommunikation erfolgt über GPRS und bringt neben E-Mail auch eine Option zur MMS-Benachrichtigung mit. Die Steuerung der Kamera erfolgt per SMS. 


\begin{tabular}{|c|c|c|c|c|c|c|c|}
\hline Hersteller & Nokia & D-Link & Panasonic & AXIS & Allnet & logiware & Kombintion \\
\hline Gerät & PT-6 & DCS-5300G & $B L-C 30$ & 212 PTZ & 2250 & go1984 pro & \\
\hline \multicolumn{8}{|l|}{ Alarmauslösung } \\
\hline Bewegungssensor & ja & nein & ja & ja & nein & nein & nein \\
\hline Videovergleich & nein & ja & nein & nein & ja & ja & ja \\
\hline Thermosensor & ja & nein & nein & nein & nein & nein & nein \\
\hline Geräuschpegelerkennung & nein & nein & nein & ja & nein & nein & nein \\
\hline Externe Eingänge & nein & nein & nein & ja & nein & unterstützt & unterstützt \\
\hline \multicolumn{8}{|l|}{ Signalisierung } \\
\hline SMS & nein & nein & nein & nein & nein & nein & nein \\
\hline MMS & ja & nein & nein & nein & nein & nein & nein \\
\hline FTP & nein & ja & ja & ja & nein & ja & ja \\
\hline E-Mail & ja & ja & ja & ja & ja & ja & ja \\
\hline \multirow[t]{2}{*}{ Externe Ausgänge } & nein & nein & nein & ja & nein & unterstützt & unterstützt \\
\hline & & & & & & \multicolumn{2}{|c|}{$\begin{array}{l}\text { zusätzlich: Soundausga- } \\
\text { be, Anruf (ISDN), Start } \\
\text { ext. Programme }\end{array}$} \\
\hline \multicolumn{8}{|l|}{ Kommunikation } \\
\hline Powerline & nein & nein & nein & nein & nein & k.A. & \\
\hline GPRS/UMTS & ja & nein & nein & nein & nein & k.A. & \\
\hline WLAN & nein & ja & ja & nein & ja & k.A. & \\
\hline Ethernet & nein & ja & ja & ja & ja & k.A. & \\
\hline \multicolumn{8}{|l|}{ Audio } \\
\hline Mikrofon intern/extern & $\mathrm{ja} /$ nein & $\mathrm{ja} / \mathrm{ja}$ & nein/nein & $\mathrm{ja} / \mathrm{ja}$ & $\mathrm{ja} / \mathrm{ja}$ & unterstützt & \\
\hline Lautsprecher intern/extern & nein/nein & nein/nein & nein/nein & nein/ja & nein/nein & k.A. & \\
\hline \multicolumn{8}{|l|}{ Zusätzl. Features } \\
\hline Fernbedienung (scharf/unscharf) & per SMS & nein & nein & nein & nein & nein & nein \\
\hline Verzögerungszeit Scharfschaltung & sofort & k.A. & k.A. & k.A. & k.A. & \multicolumn{2}{|c|}{ programmabhängig } \\
\hline Keepalivesignal & nein & nein & nein & nein & nein & nein & nein \\
\hline Nachtsichtfunktion & ja & nein & nein & nein & nein & nein & nein \\
\hline Bewegungssteuerung der Kamera & nein & ja & ja & ja & ja & unterstützt & unterstützt \\
\hline Webserver auf Kamera integriert & nein & ja & ja & ja & ja & k.A. & ja \\
\hline Videostreaming & nein & ja & ja & ja & ja & k.A. & ja \\
\hline Preis & $299,99 €$ & $405,90 €$ & $239,90 €$ & $549,00 €$ & $179,90 €$ & $349,00 €$ & $528,90 €$ \\
\hline
\end{tabular}

Tab. 1: Detailvergleich der Geräte (alle Geräte sind in der kompl. Belegarbeit zu finden), Anm: Preise, Stand November 2006

\section{Technische Planung}

Immer mehr Haushalte in Deutschland sind von Einbrüchen betroffen. Um sich davor zu schützen bzw. das Sicherheitsgefühl zu erhöhen, benötigen diese Haushalte Überwachungssysteme. Diese sind meist teuer und müssen fest installiert werden, was in einer Mietwohnung zu Problemen führen kann. An dieser Stelle setzt der Grundgedanke des neuen Kamera Überwachungssystems Argus von T-Systems an. Argus soll kostengünstig, flexibel, leicht bedienbar, ohne großen Aufwand zu montieren sein und trotzdem einen hohen Funktionsumfang bieten.

\subsection{Systemkonzept}

Argus ist ein »Rund um die Uhr «-Überwachungssystem für jegliche Objekte, die überwacht werden sollen. Voraussetzung für die Nutzung sind ein Breitband-Internetanschluss und ein Computer zum Konfigurieren des Systems. Das Endgerät besteht aus einer Digitalkamera mit einer Auflösung von 640 x 480 Pixeln, einem integrierten, passiven Infrarotbewegungsmelder, integrierten Infrarot-LEDs für die Nachtsichtfunktion, einer Alarm- sirene und einer Fernbedienung. Die Verbindung zum Internet kann entweder über ein RJ45-Netzwerkkabel direkt am DSL-Router realisiert werden oder kabellos per WLAN-Funktionalität.

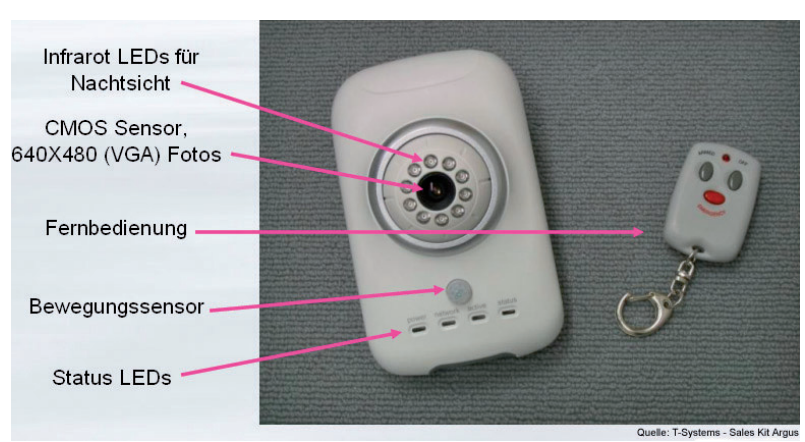

Abb. 1: Argus-Überwachungssystem inkl. Fernbedienung

Das Überwachungssystem stellt nach Anschluss des Routers und der Stromversorgung eine Verbindung zum Eventmanager her, der über eine Schnittstelle zum Secure Home Center von T-Systems verfügt. Nach dem ersten Anmelden des Sicherheitssystems beim Eventmanager sendet es im Minutentakt ein »Statussignal«, um die 
Funktionsbereitschaft zu signalisieren. Wird dieses Signal nicht mehr gesendet, ist von einem Alarm auszugehen. Kommt es dazu bzw. schlägt der Bewegungsmelder an, wird vom Eventmanager das Secure Home Center von TSystems benachrichtigt. Über eine Web-Oberfläche kann die Kamera vom Nutzer konfiguriert werden. Verschiedene Einstellungen, wie Alarmprofile, Benachrichtigungen bei Alarm und WLAN Sicherheitseinstellungen, können vom Nutzer vorgenommen werden. Des Weiteren ist es möglich, die Bilder von vergangenen Alarmen auszuwerten und weiter zu verarbeiten.

Der Nutzer benötigt zum Betreiben des Überwachungssytems einen DSL-Breitbandanschluss. Bilder der Überwachungskamera und Daten des Bewegungsmelders werden an das Alarm Monitoring Center von T-Systems übertragen. Dort ist es dem Nutzer möglich, aktuelle Informationen aus seiner Wohnung abzurufen. Registriert das Alarm Monitoring Center einen Alarm, wird dieser über einen vom Nutzer ausgewählten Kanal an ihn weitergeleitet. Durch die Benachrichtigung entsteht weiterer Traffic auf dem entsprechenden Kanal. Für beide Vertragsparteien entstehen Vorteile. Das bedeutet für den Nutzer des Systems ein höheres Gefühl von Sicherheit bei ständigem Einblick in seine Wohnung. Der Betreiber profitiert am Verkauf der Geräte (ca. 350 Euro/ St.), an der monatlichen Grundgebühr von ca. 5 Euro, an der Erzeugung von (Netz-)Verkehr auf den Benachrichtigungskanälen, wie SMS und MMS, und als Anbieter von Breitband-Internetzugängen.

\subsection{Systemkonzept UMTS-Variante}

Neben dem bereits beschriebenen Argus-System mit Breitband-Internetanschluss (Variante 1) soll an dieser Stelle eine zweite Möglichkeit zur Realisierung aufgezeigt werden. Dabei werden die Daten des Überwachungssystems via UMTS an das Alarm Monitoring Center übertragen. Der Funktionsumfang des Systems wird dadurch nicht beeinflusst oder verändert. Der einzige Unterschied liegt im Übertragungsweg der Daten. Das Überwachungssystem sendet seine Daten via UMTS an den Betreiber. Der Mobilfunkanbieter kann dafür ggf. eine Grundgebühr und das Volumen der Daten berechnen, also ähnlich der Abrechnung eines Breitbandanschlusses eines Festnetzanbieters. Der Nutzer erhält, wie auch bei Variante 1, ein vollwertiges Überwachungssystem. Der große Unterschied liegt darin, dass durch den mobilen Breitbandanschluss jegliche Installationsarbeiten für den Nutzer entfallen, wodurch das Einrichten des Systems leicht zu bewältigen ist. Der Betreiber des Systems profitiert, wie auch bei Variante 1, von dem Verkauf der Geräte und dem erzeugten Verkehr durch die Benachrichtigung bei einem Alarm.

\subsection{Kostenanalyse}

Nachdem die stärksten Wettbewerber des Argus-Systems ermittelt wurden, soll das Produkt mit der besten Performance im Bereich Mobilfunk (Nokia PT-6) mit dem Argus-UMTS-Überwachungssystem verglichen werden.
Es stellt sich die Frage, ob ein solches System überhaupt rentabel ist. Als Referenz dient das Argus-System mit Breitband-Internetzugang. Betrachtet werden die Betriebskosten und eine definierte Anzahl von ausgelösten Alarmen. Für dieses Rechenbeispiel wird davon ausgegangen, dass pro Monat zehn Benachrichtigungen per MMS an den Nutzer gesendet werden. Dafür hat der Nutzer monatlich Betriebskosten laut Tab. 2 einzuplanen.

\begin{tabular}{|l|r|r|r|}
\hline & Argus & \multicolumn{1}{|l}{$\begin{array}{l}\text { Argus } \\
\text { UMTS }\end{array}$} & $\begin{array}{l}\text { Nokia } \\
\text { PT-6 }\end{array}$ \\
\hline Grundgebühr für das System & $4,95 €$ & $4,95 €$ & keine \\
\hline $\begin{array}{l}\text { Grundgebühr Festnetz- } \\
\text { anschluss (anteilig) }\end{array}$ & $10,00 €$ & keine & keine \\
\hline $\begin{array}{l}\text { Breitband-Internetanschluss } \\
\text { (anteilig) }\end{array}$ & $10,00 €$ & keine & keine \\
\hline $\begin{array}{l}\text { Grundgebühr SIM-Karte } \\
\text { inkl. 30MB }\end{array}$ & keine & $9,90 €$ & $9,90 €$ \\
\hline 10x MMS Kosten & $44,75 €$ & $34,65 €$ & $9,90 €$ \\
\hline Gesamt: & & & keine \\
\hline
\end{tabular}

Tabelle 2: Kostenvergleich

Da das Nokia-System nur MMS bzw. kostenfreie E-Mails versenden kann, wird beim Vergleich mit dem ArgusSystem die MMS-Funktionalität herangezogen. Es ist nicht eindeutig bestimmbar, ob die $30 \mathrm{MB}$ Freivolumen bei der Argus-UMTS-Version ausreichen, da die Daten nicht direkt an den Empfänger, sondern an das Event Center sendet. Festzustellen ist aber, dass die Mobilfunksysteme eindeutig günstiger sind als die Systeme mit einem Festnetzanschluss. Das liegt zum einen an den hohen Kosten für einen Breitbandanschluss (wurde nur anteilig berechnet) und zum anderen an der Einzelabrechnung der gesendeten MMS an den Nutzer. Für die Mobilfunkversionen gibt es von allen großen Mobilfunkanbietern Volumentarife, die mit GPRS oder UMTS genutzt werden können. Der Kostenvergleich zeigt aber auch, dass das System von Nokia im Preiskampf schwer zu schlagen sein wird. Es bietet zum einen den Vorteil, dass keine monatliche Grundgebühr für das System zu zahlen ist, und zum anderen, dass die gesendeten MMS in der Grundgebühr des bezahlten Datenvolumens enthalten sind. Das heißt, bei dem Nokia-System werden nur die tatsächlich entstanden MMS ohne Aufschlag abgerechnet. Beim Argus-System ist dies nicht der Fall. Da die MMS vom Service Center versandt werden, sind diese nicht über die Datenkarte in der Kamera abrechenbar und müssen einzeln bezahlt werden.

\section{Geschäftliche Planung und Umsetzung}

\subsection{Marktanalyse}

Durch den Verkauf von Sicherheitssystemen will T-Systems nicht nur am Markt für Sicherheitstechnik Fuß fassen, sondern auch Partnerunternehmen die Chance geben, sich am Markt für Breitband-Internetzugänge 
weiter zu profilieren. Neben der Betrachtung dieser beiden Märkte wird als dritter Markt der Mobilfunkmarkt untersucht, um die Chancen für eine Argus UMTS Version zu prüfen.

\subsubsection{Sicherheitsmarkt}

Der Markt für Sicherheitstechnik erlebt zur Zeit einen Umschwung. Bis vor einigen Jahren wurden nur fest installierte »High-End «Alarmsysteme verkauft. Die Kosten dafür waren enorm hoch und eine Fernüberwachung nur schwer zu realisieren. Mit dem immer größer werdenden Sicherheitsbedürfnis der Menschen entschieden sich die Hersteller von Sicherheitsprodukten für die Entwicklung neuer, kostengünstigerer Lösungen.

Die Erwartungen der Kunden an ein solches System sind: einfache Handhabung, kostengünstige Anschaffung und Betreibung, schnelle Reaktion auf Ereignisse, effektiver Schutz, Möglichkeit der Fernsteuerung und ein vertrauenswürdiges Unternehmen als Ansprechpartner.

Statistiken zeigen, dass die Gefahr, Opfer eines Wohnungseinbruchs zu werden, heute viermal größer ist als vor 30 Jahren. Allein im Jahr 2006 wurden 130.055 Einbrüche in Deutschland erfasst. Durch Befragungen und Marktstudien zeigte sich, dass die Ängste vor einem Verbrechen besonders in Deutschland steigen. 50 Prozent der befragten Haushalte sind über einen möglichen Einbruch besorgt. 33 Prozent davon befürchten einen Einbruch in den nächsten 12 Monaten.

\subsubsection{Markt der Breitband-Internetzugänge}

Ähnlich dem Sicherheitsmarkt ist auch der Markt für Breitband-Internetzugänge ein stark wachsender Markt. Auch dieser Markt ist vom harten Konkurrenzkampf um potenzielle Kunden gezeichnet, wobei der maßgebliche Entscheidungsgrund für einen Anbieter der Preis ist. Um einen erhöhten Preis rechtfertigen zu können, müssen die Provider Zusatzdienste anbieten bzw. nach Möglichkeiten suchen, um sich von der Konkurrenz abzusetzen. Ein Ende des DSL-Booms ist nach Meinung des BITCOM-Präsidenten Willi Berchtold nicht absehbar. So soll es Ende 2006 bis zu 15 Millionen DSL-Anschlüsse in Deutschland geben, das würde einem Wachstum von ca. 30 Prozent entsprechen.

\subsubsection{Mobilfunkmarkt}

Auf dem deutschen Mobilfunkmarkt finden zwei Kopfan-Kopf-Rennen statt. Während die großen Anbieter T-Mobile und Vodafone um die Marktführerschaft kämpfen, liefern sich $\mathrm{O}_{2}$ und E-Plus ein Rennen um den dritten Platz am Markt. Doch im Gegensatz zu den beiden zuvor beschriebenen Märkten ist der Mobilfunkmarkt relativ gesättigt. Das bedeutet, es gibt immer weniger potentielle Neukunden. Nach Angaben der Regulierungsbehörde für Telekommunikation und Post haben in Deutschland über 80 Prozent der Bevölkerung ein Mobiltelefon. Daher geht der Wettbewerb vom Kampf um die Neukunden jetzt in einen harten Verdrängungswettbewerb über. Aus diesem Grund konzentrieren sich die Anbieter zur Zeit vor allem auf zwei Dinge. Sie wollen erstens die Kunden gewinnen, die noch kein Mobiltelefon haben, aber einen guten Umsatz versprechen, und sie wollen zweitens ihre umsatzstarken Kunden halten. Mit dem Aufkommen von immer schnelleren Übertragungstechniken, wie GPRS, EDGE und UMTS, nimmt auch der Marktanteil für Datendienste stark zu. Bei einem geschätzten NonVoice-Anteil von 20,7 Prozent im Mobilfunkmarkt fielen davon im Jahr 2006 allein 6,1 Prozent auf Datendienste zurück. Das entspricht fast einer Verdopplung gegenüber dem Vorjahr 2005.

\subsection{Berechnung des Geschäftsmodells Argus-UMTS}

Für die Berechnung des Geschäftsmodells müssen zuerst einige Annahmen definiert werden, um folgende Aussagen zuordnen zu können. Das Geschäftsmodell wird für T-Systems berechnet. Es wird für eine Zeitspanne von fünf Jahren betrachtet. Die Hardware der Geräte wird komplett bei einem Zulieferer eingekauft. Das heißt, für die Entwicklung der Hardware wird aus der Sicht von T-Systems kein Aufwand getrieben. Es wird lediglich ein fester, vereinbarter Betrag pro Gerät bezahlt. Den Aufwand für die Entwicklung des Servicesystems übernimmt T-Systems selbst. Verkauft werden die Überwachungssysteme an Vertriebspartner, wie z. B. T-Online, welcher die Geräte an den privaten Endkunden weiterverkauft.

T-Systems bleibt somit nur Betreiber des Servicesystems der Überwachungssysteme und betreibt den thirdlevel support für spezielle Fragen zum System gegenüber dem Endkunden. Alle verwendeten Zahlen in der Berechnung des Geschäftsmodells wurden nach bestem Wissen geschätzt und bauen auf aktuellen Zahlen von T-Systems auf.

\subsubsection{Personal}

Der erste Ansatz zur Berechnung des Geschäftsmodells ist die Planung der Mitarbeiter in dem Projekt Argus. Für die Zeit der Entwicklung des Service Centers ist ein Personalbedarf von acht Mitarbeitern geplant. Die Entwicklungszeit sollte nach sechs Monaten abgeschlossen sein. Nach dieser Zeit sollte das Service Center in den normalen Betrieb übergehen. Zwei von ehemals drei Entwicklern sollten das Service Center weiter betreuen. Für die Aufgaben des third level supports sollten nun drei statt ehemals ein Mitarbeiter eingesetzt werden, da mit Verkaufsbeginn des Systems mehr Bedarf an Support aufkommt. Daraus resultiert in der Entwicklungszeit eine monatliche Belastung von ca. 39.000 Euro. Im Normalbetrieb fallen durch den größeren Aufwand beim Support monatlich ca. 43.000 Euro an.

\subsubsection{Investitionsplan}

Im Investitionsplan werden die Kosten festgehalten, die zu Beginn des Projektes Argus-UMTS einmalig getätigt werden müssen. Dabei handelt es sich um Kosten der Betriebs- und Geschäftsausstattung, Marketingkosten, Kosten für das Warenlager und Kosten für die Forschung und Entwicklung. Für die vorwettbewerbliche 
Forschungs- und Entwicklungsleistung wurden sechs Monate eingeplant. Die Gesamtinvestitionskosten belaufen sich auf ca. 600.000 Euro.

\subsubsection{Herstellungskosten und Verkaufszahlen}

Durch den Einkauf der kompletten Hardware des UMTSSicherheitssystems bei einem Zulieferer entstehen TSystems keine direkten Herstellungskosten. In Eigenentwicklung wird allerdings das Service Center erstellt. Diese Kosten werden, wie auch andere betriebsinterne Kosten, in die Herstellungskosten mit einbezogen. So errechnet sich ein Verkaufspreis von 301 Euro je Gerät.
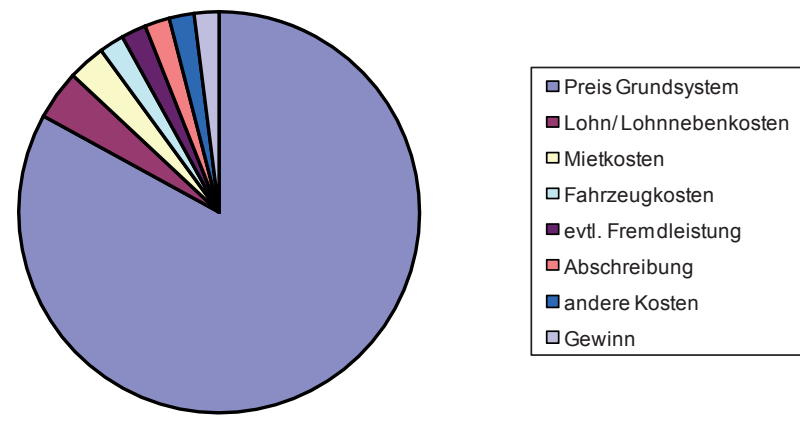

Abb. 2: Kostenzusammensetzung Argus-UMTS

Zusätzlich zum Verkaufspreis eines Sicherheitssystems erhält T-Systems eine monatliche Grundgebühr in Höhe von 4,95 Euro je Gerät für die Betreibung des Service Centers. Die Verkaufszahlen der Geräte können nur geschätzt werden. Da als Anbieter für den Endkunden T-Online in Frage kommt, kann die Schätzung relativ genau durchgeführt werden. Wie bei der ArgusBreitband-Version bringt T-Online Geräte nur in den Handel, wenn sicher ist, dass drei Prozent der Kunden in zwei Jahren damit erreicht werden. Das sind bei 11 Millionen T-Online Kunden 330.000 Geräte. Nimmt man zusätzlich noch an, dass die UMTS Sicherheitssysteme nicht nur von T-Online, sondern von einem weiteren Service Provider vertrieben werden, kann die Zahl noch leicht erhöht werden. Bei unserer Berechnung sind wir nach einer Einführungsphase am Markt von jährlich ca. 250.000 verkauften Systemen ausgegangen.

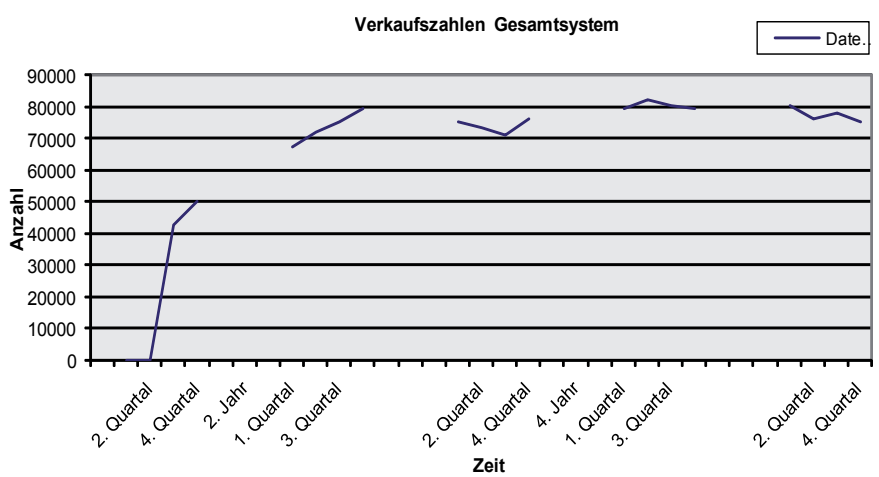

Abb. 3: Verkaufszahlen Argus UMTS über 5 Jahre

\subsubsection{Gewinn- und Verlustrechnung}

Nach Berechnung der definierten Werte kann schon nach dem ersten Jahr ein positives Betriebsergebnis ausgewiesen werden. Ein Zinsaufwand aus Krediten wird nicht einbezogen, da davon ausgegangen wird, dass die Finanzierung aus betriebseigenen Mitteln realisiert wird. Wie bereits angedeutet, beruhen wichtige Werte, wie Verkaufszahlen, Abschreibungen u. v. m., auf Schätzungen, so dass die Gewinn- und Verlustrechnung nur eine grobe Richtung vorgeben kann.

\subsubsection{Liquiditätsplanung}

Die Liquiditätsplanung ist für ein Unternehmen besonders wichtig, da hier zu erkennen ist, ob die finanziellen Mittel für das zu untersuchende Geschäftsmodell ausreichen. Aus Sicherheitsgründen wird bei der Berechnung davon ausgegangen, dass sich Zahlungseingänge der Kunden nicht sofort einstellen. Das heißt, wird ein Sicherheitssystem verkauft, kann T-Systems erst im darauf folgenden Monat mit dem Geld und der Grundgebühr rechnen. Dieser Fall muss nicht eintreten, ist aber denkbar. Berechnet man die Liquidität mit einem Monat Verzug, also kaufmännisch konservativ, ergibt sich folgende Situation:

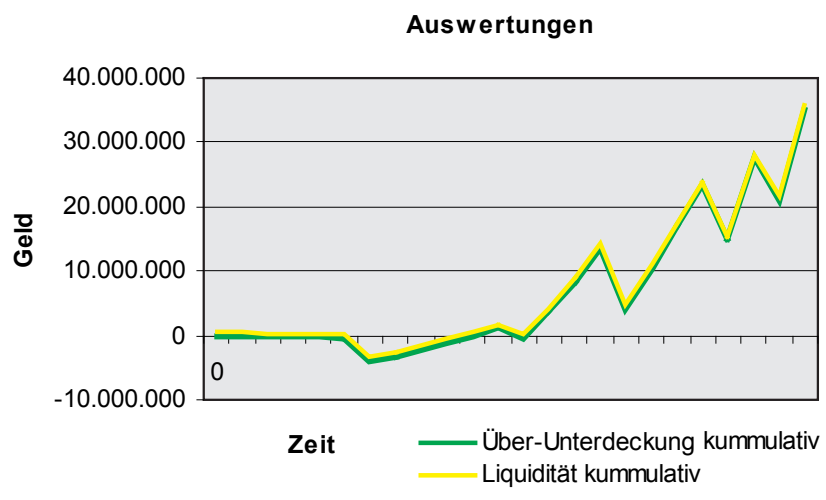

Abb. 4: Liquiditätsplanung Argus-UMTS

Sobald die Entwicklungsphase abgeschlossen ist und die ersten Sicherheitssysteme verkauft werden, rutscht die Liquidität ins Negative ab, da die geplanten Einnahmen erst einen Monat später zur Verfügung stehen. Diese Tendenz muss nicht auftreten, wenn der Kunde sofort zahlt. Tritt allerdings der Fall ein, stehen dem Geschäftsmodell keine finanziellen Mittel (mehr) zur Verfügung. Besteht eine solche Situation, sind dafür entsprechende Gegenmaßnahmen einzuleiten. Es könnte weiteres Kapital aus dem Unternehmen benötigt werden oder die Finanzierung müsste überdacht werden. Nach der Pufferung dieser kritischen Phase entwickelt sich die Liquidität hervorragend. Der Break Even Point wird im Monat elf des ersten Geschäftjahres durchbrochen, was dem fünften Verkaufsmonat entspricht. 


\section{Fazit}

Bei den ersten Recherchearbeiten nach ähnlichen Systemen war es anfangs schwer, geeignete Produkte zu finden. Das lag allerdings nicht daran, dass diese nicht vorhanden waren, sondern der Bekanntheitsgrad einfach fehlte. Genau dieses Problem könnte sich Argus zum Vorteil machen. Die gezielte Werbung als Sicherheitssystem muss das Bedürfnis des Käuferkreises zuerst wecken. Bisher scheint es so, als würden die Menschen nicht wissen, was es auf dem Markt der Low Cost-Sicherheitssysteme bereits gibt.

Bei dem Vergleich von Argus mit anderen Systemen fiel auf, dass jedes System seine Vor- und Nachteile hat. So kann Argus besonders mit einem Keep-alive-Signal und einer Fernbedienung punkten. Sehr nachteilig wirkt sich die monatliche Grundgebühr von 4,95 Euro aus. Für kein anderes auf dem Markt verfügbares System müssen Grundgebühren gezahlt werden. Gute Systeme haben einen Webserver mit dynamischer IP-Adresse integriert und sind dadurch, genau wie das Argus-System, zu erreichen. Das für den Nutzer so wichtige, leichte Installieren des Systems wird von Argus nur bedingt erfüllt. Es müssen Netzwerkeinstellungen vorgenommen werden, mit denen viele Personen voraussichtlich überfordert sind. Weiterhin ist aufgefallen, dass zur Nutzung der WLANFunktionalität die kompletten WLAN-Sicherheitsdaten mit Schlüssel auf einer Internetseite bekannt gegeben werden müssen.

In wieweit der Nutzer diese vertraulichen Daten weitergeben möchte, sollte geprüft werden. Ein Vorbild für die Einfachheit der Installation ist das Gerät PT-6 von Nokia. Dieses System kann wirklich »jedermann « montieren. Die Argus-UMTS-Lösung ist eine sehr interessante Alternative. Mit ihr könnte ein leichtes Handhaben realisiert werden (ähnlich PT-6) und der Aufstellort der Kamera wäre flexibler. Die Einrichtung der Kamera auf einem evtl. Netzwerk würde komplett entfallen, was auch die Nutzung eines Computers überflüssig macht. Wie in der Kostenanalyse gezeigt, würden sich auch die Kosten für dieses System besser vermarkten lassen.

\section{Danksagung}

Für die Unterstützung und die gute Zusammenarbeit bedanken wir uns vielmals bei Professor Dr. Bernd Eylert. Ohne sein Engagement wäre diese Arbeit nicht möglich gewesen. Weiterhin bedanken wir uns für die gute $\mathrm{Zu}$ sammenarbeit bei Dr. Reiner Krämer von T-Systems für das Thema.

\section{Literatur und Internetquellen}

Wirtz, B. W., Electronic Business, Wiesbaden 2001

http://www.t-systems.de/

http://www.haus-und-markt.de/artlist.php4?rubrikid=7

http://www.dsltarife.net/

\section{Autoren}

Christian Halama, M. Eng.

Technische Fachhochschule Wildau

Studiengang Telematik

Schillerallee $1 / 415$

15745 Wildau

mail@christianhalama.de

Nico Venske, M. Eng.

Technische Fachhochschule Wildau

Studiengang Telematik

Fichtestr. 4

15711 Königs Wusterhausen

n.venske@gmx.de 